\title{
Muslime in Europa zwischen Globalisierung und Lokalisierung - Gesellschaftspolitische und theologische Perspektiven im Anschluss an Enes Karic und Tariq Ramadan*
}

\author{
Hansjörg Schmid
}

\section{Vorbemerkungen: Der Globalisierungsdiskurs und Muslime in Europa}

Muslime finden in Deutschland und Westeuropa in den letzten Jahren eine zuvor nicht gekannte Beachtung. Es waren in erster Linie außereuropäische Ereignisse - angefangen mit der Revolution im Iran im Jahr 1979 bis hin zum 11. September 2001 -, die zu einer intensiven Wahrnehmung der muslimischen Minderheiten in Europa geführt haben. In der Konsequenz wurden Gruppen, die zuvor meist ethnisch als Araber oder Türken qualifiziert wurden, erst als Muslime wahrgenommen. Diese Entwicklung hängt unmittelbar mit der Globalisierung zusammen, die zu einer erhöhten Aufmerksamkeit für lokale Phänomene führt. Damit ist auch die Wechselwirkung zwischen Globalem und Lokalem bereits angedeutet.

Das Thema „Muslime und Globalisierung“ wurde bislang meist im Zusammenhang mit der islamischen Welt und der Debatte um Verwestlichung diskutiert. ${ }^{1}$ Dort gibt es zwar ganz verschiedene

\footnotetext{
* Ich danke Prof. Dr. Hans Zirker für zahlreiche Anregungen und kritische Hinweise zu diesem Beitrag.

1 Vgl. z.B. Schreiter, R. J., Globalisierung, Postmoderne und die neue Katholizität, in: ÖR 53 (2004) 139-159, 145; Fürtig, H., Muslime in der Globalisierung. Wahrnehmungen und Reaktionen, in: ders. (Hg.), Islamische Welt und Globalisierung. Aneignung, Abgrenzung und Gegenentwürfe, Würzburg 2001, 17-50; Renz, Andreas, Globalisierung - Muslimische Wahrnehmungen und Reaktionen, in: Ozankom, C./ Udeani, C. (Hg.), Globalisation - Cultures

- Religions. Globalisierung - Kulturen - Religionen, Amsterdam/New York 2006,
} 
Reaktionen: Dabei überwiegen jedoch klar negative Positionen. ${ }^{2}$ So wird Globalisierung in der islamischen Welt vielfach als Fortsetzung des Kolonialismus gesehen und als Fremdbestimmung erfahren. Hier soll der Fokus auf Europa liegen, dessen Muslime mit Globalisierung möglicherweise anders umgehen als die Muslime in der islamischen Welt, die aber möglicherweise auch Reaktionsmuster aus der islamischen Welt importieren. Es geht dabei um die beiden folgenden Leifragen:

(a) Wie sind die Phänomene Globalisierung und Lokalisierung wirksam für Muslime in Europa? (siehe dazu 2.-4.)

(b) Welche theologischen Deutungen (evtl. mit Aufgreifen des Begriffs) ziehen sie nach sich? (siehe dazu 5.)

Die spezifischen Antworten und Reaktionen, die Muslime unter europäischen Rahmenbedingungen entwickeln, sind von nicht $\mathrm{zu}$ unterschätzender Bedeutung, denn auch sie wirken ihrerseits wiederum im Duktus der Globalisierung auf die islamische Welt zurück, wenngleich bisher meist die umgekehrte Bewegungsrichtung untersucht wurde.

„Muslime in Europa“ sind keine einheitliche Größe. Im Gegenteil stellen sie eine einzigartige Vielfalt dar: Großteils sind die Muslime in Europa Migranten aus verschiedenen Teilen der Welt, deren „mitgebrachte“ Herkunftskulturen durch die Begegnung mit europäischen Kulturen transformiert werden. ${ }^{3}$ Der dadurch

81-109 (dort auch weitere Literatur). Beyer, Peter, Globalisierung und Religion, in: ders. (Hg.), Religion im Prozeß der Globalisierung, Würzburg 2001, XI-LVII, XIV sieht in „Globalisierung“ einen Begriff, „der über das Konzept der Modernisierung hinauszugehen versucht, ohne seine Kontinuität mit diesem zu leugnen“.

2 Vgl. Riddell, P.G., Christians and Muslims. Pressures and Potential in a Post9/11 World, Leicester 2004, 103, und Wielandt, Rotraud, Kulturelle Pluralität und Globalisierung in der Sicht zeitgenössischer muslimischer Intellektueller, in: JCSW 41 (2000) 74-86, 75: „Unter zeitgenössischen Muslimen - gleich welcher Observanz - besteht weithin Einigkeit darüber, dass sich die ganze Welt auch nach dem Ende der Kolonialherrschaft europäischer Staaten unter der nicht zuletzt ökonomisch motivierten kulturellen Hegemonie des Westens befindet.“ Als ein Beispiel vgl. Amin, Samir, Die Zukunft der Weltsysteme, Hamburg 1997.

3 Eindrucksvoll dargestellt wird diese Vielfalt bei Waardenburg, J., Muslims and Others. Relations in Context, Berlin/New York 2003, 336f. 
entstehenden innerislamischen Vielfalt ist hier in besonderer Weise Rechnung zu tragen: Positionen zur Globalisierung sind immer nur exemplarisch; auch bei den folgenden Überlegungen kann immer nur ein kleiner Ausschnitt aus dieser Vielfalt dargestellt werden. Da die innermuslimischen Diskussionsprozesse in Europa noch in den Anfängen stecken, ist auch unsicher, welche Deutung sich mehrheitlich durchsetzen wird. Schwerpunkt meiner persönlichen Wahrnehmungen und Beobachtungen ist der deutschsprachige Raum, aus dem bislang jedoch weniger profilierte muslimische Stimmen kommen als aus anderen europäischen Ländern. Exemplarisch möchte ich mich daher in den folgenden Überlegungen vor allem auf zwei nicht aus Deutschland stammende muslimische Denker beziehen, in deren Werk die Frage der Globalisierung einen zentralen Raum einnimmt und die sich bereits aufgrund ihrer Herkunft und Biographie stark unterscheiden: ${ }^{4}$

Tariq Ramadan wurde 1962 in Genf als Sohn des ins Schweizer Exil geflohenen Said Ramadan geboren, der Schüler und Schwiegersohn des Gründers der ägyptischen Muslimbrüder, Hasan al-Banna, war. Ramadan studierte zunächst französische Literatur und Philosophie. U.a. nach einem Studienjahr in Ägypten, wo er Islamwissenschaften studierte, wurde er in Genf über Hasan al-Banna promoviert. Ramadan repräsentiert den gebildeten Migrationsislam. Er ist als Gastprofessor und populärer Prediger mit großer Breitenwirksamkeit durch eine große Zahl von Schriften und Vortragsmitschnitte bekannt. $^{5}$ Aufgrund seiner Popularität vor allem im französischen

\footnotetext{
4 Mit einem Exponenten des arabischen und einem des bosnischen Islam in Europa sind zwei wichtige Traditionsströme erfasst. Für die türkische Diskussion, die hier aus Platzgründen nicht berücksichtigt werden kann, vgl. Kuru, A.T., Globalization and Diversification of Islamic Movements. Three Turkish Cases, in: Political Science Quarterly 120 (2005), 253-274; Keyman, E. F., Globalization, Civil Society and Islam. The Question of Democracy in Turkey, in: Jenson, J./ de Sousa Santos, B. (Hg.), Globalizing Institutions. Case Studies in Regulation and Innovation, Aldershot 2000, 207-231. Weitere zu berücksichtigende Autoren wären Yasin A. und Mehmet A.

5 Vgl. zur Person und Biographie Ammann, Ludwig, Tariq Ramadan, Schweiz/Frankreich. Der konservative Reformer, in: Amirpour, K./Ammann, L. (Hg.), Der Islam am Wendepunkt. Liberale und konservative Reformer einer Weltreligion, Freiburg 2006, 23-33, und Ghadban, Ralph, Tariq Ramadan und die
} 
Sprachraum kann Ramadan als eine der Leitfiguren des Islam in Europa gelten.

Enes Karic wurde 1958 in Bosnien als Sohn eines Imams geboren. Er hat Islamwissenschaften und Philosophie studiert und wurde über hermeneutische Probleme bei der Übersetzung des Korans ins Serbokroatische promoviert. Derzeit ist er Professor für Koranwissenschaften und Dekan der islamisch-theologischen Fakultät in Sarajevo. Er war 1994-1996 Erziehungs- und Kulturminister. Karic entstammt dem autochthonen Islam auf dem Balkan und kann als Vordenker des bosnischen Islam angesehen werden. ${ }^{6}$

Islamisierung Europas, Berlin 2006 (zur Charakterisierung Ramadans vgl. v.a. 4245). Vgl. auch Wrogemann, Henning, Missionarischer Islam und gesellschaftlicher Dialog. Eine Studie zur Begründung und Praxis des Aufrufs zum Islam, Frankfurt 2006, 348-355; Zemouri, Aziz, Faut-il faire taire Tariq Ramadan?, Paris 2005; Valkenberg, P., Sharing Lights on the Way to God. Muslim-Christian Dialogue and Theology in the Context of Abrahamic Partnership, Amsterdam/New York 2006, 321-327. - Im Folgenden gehe ich von zwei Hauptwerken Ramadans (Ramadan, Tariq, Muslimsein in Europa. Untersuchung der islamischen Quellen im europäischen Kontext, Marburg 2001; Ramadan, Tariq, Western Muslims and the Future of Islam, Oxford/New York 2004) und einigen kleineren Beiträgen aus. Natürlich ist damit zu rechnen, dass die unterschiedlichen Kontexte der Publikationen zu unterschiedlich gewichteten inhaltlichen Positionen führen. Hier geht es jedoch um die Darstellung von Grundlinien seines Denkens, weshalb ich eine synchrone Lektüre der Werke Ramadans durchführe.

6 Vgl. zur Person und Biographie Bougarel, X., Trois définitions de l'islam en Bosnie-Herzégovine, in: Archives de Sciences Sociales des Religions 115 (2001), 183-201 (http://www.ehess.fr/centres/ceifr/assr/N115/ 009.htm - Seitenzählung nach dieser Fassung), 4; Karic, E., Essays (on Behalf) of Bosnia, Sarajevo 1999, $290 \mathrm{f}$. und http://www.fin.ba/fin_info/bibliografije/enes_karic.htm. Zum Hintergrund vgl. Karcic, F., The Bosniaks and the Challenges of Modernity. Late Ottoman and Hapsburg Times, Sarajevo 1999, 109-159. Seit der Habsburgerzeit war durch die Einbindung des Islam eine „Doppelbildung“ muslimischer Funktionsträger in islamischen Wissenschaften und europäischem Recht etc. erforderlich (vgl. 138). Dies schlägt sich bis heute an der islamischen Fakultät in Sarajevo und auch bei Karic, E. nieder. - Den beiden folgenden Untersuchungen liegen zwei Büchern von Karic (E. Karic, Land; Karic, E., Essays (on Behalf) of Bosnia, Sarajevo 1999) zugrunde, bei

denen es sich um Sammelbände mit ursprünglich selbständig veröffentlichten Aufsätzen handelt. Dennoch werden die Bücher als Einheit betrachtet und der Einfachheit halber auch als Monographien zitiert. 


\section{Grundthese: Dynamik zwischen Globalisierung und Lokalisierung}

Globalisierung ist ein ideologisch aufgeladener Leitbegriff der aktuellen Debatte. Der Begriff der Lokalisierung ist demgegenüber weit weniger verbreitet. Im Folgenden wird die Grundthese vertreten, dass die beiden zunächst gegensätzlichen Phänomene zusammengehören und sich gegenseitig bedingen. ${ }^{7}$ Wenn im Titel dieses Abschnitts wie auch des gesamten Beitrags von „Zwischen“ die Rede ist, meint dies nicht Passivität. Auch Muslime sind Akteure der aktuellen Entwicklungen, die sich dynamisch zwischen beiden Phänomenen bewegen.

Da Globalisierung kein einheitlicher Begriff ist, ${ }^{8}$ muss zunächst kurz beschrieben werden, was darunter verstanden werden soll: Es handelt sich um Entwicklungen auf der Basis von modernen Datenverarbeitungs- und Kommunikationssystemen sowie des Transportwesens. Dies ermöglicht nicht nur zunehmende wirtschaftliche Verflechtungen, sondern auch Migration, Tourismus und die fast grenzenlose Möglichkeit zum Informationsaustausch. Räumliche Distanzen verlieren an Bedeutung. Wissen ist über Grenzen hinweg durchs Internet verfügbar. Staatenübergreifende und zwischenstaatliche Zusammenhänge werden mehr und mehr wirksam; transnationale Konzerne gewinnen an Einfluss. Globalisierung bedeutet somit in vielfacher Hinsicht eine Entgrenzung: „Globalisierung meint das erfahrbare Grenzenloswerden alltäglichen Handelns in den verschiedenen Dimensionen der Wirtschaft, der

7 Vgl. Schreiter, R. J., Die neue Kontextualität. Globalisierung und Fragmentierung als Herausforderung an Theologie und Kirche, in: JbM 31 (1999) 2949, 37; Beck, U., Was ist Globalisierung? Irrtümer des Globalismus - Antworten auf Globalisierung, Frankfurt 1999, 81, 90; Ayish, M. I., Arab World

Television in the Age of Globalization. An Analysis of Emerging Political, Economic, Cultural and Technological Patterns, Hamburg 2003, 69f.; H. Fürtig, Muslime 41.

8 Vgl. Beck, U., Globalisierung 44; Schreiter, R.J., Die neue Katholizität.

Globalisierung und die Theologie, Frankfurt 1997, 20. Auch zum Folgenden vgl.

Beck, U., Globalisierung passim; Kaufmann, F.-X., Art. Globalisierung, I.

Grundlegung, in: LThK3, Ergänzungsband, 95-98. 
Information, der Ökologie, der Technik, der transkulturellen Konflikte und Zivilgesellschaft [...].“9 Globalisierung ist zum einen ein deskriptiver Begriff für die beschriebenen Entwicklungen, kann aber in der Konsequenz auch zu einer Ideologie mit stark neoliberalen Zügen werden. ${ }^{10}$

Muslime sind unmittelbar von der Globalisierung betroffen: ${ }^{11}$ Ihre Präsenz in Westeuropa steht in Zusammenhang mit Wanderungsbewegungen des 20. Jahrhunderts, die weltweit zu einem intensiveren Kontakt zwischen verschiedenen Kulturen führen. ${ }^{12}$ Diese Situation ist auch Ausgangspunkt für die Schriften der beiden hier untersuchten Denker: Ramadan geht von einer offenen Welt ohne klare Grenzen aus, in der die Muslime verstreut leben und die sie neu vor die Frage stellt, sich gegenüber der nichtislamischen Umgebung zu positionieren. ${ }^{13}$ Karics Ausgangspunkt ist zwar die Präsenz der Muslime in Bosnien seit Jahrhunderten. Von da ausgehend blickt er jedoch auf die neue Situation der Muslime im Westen. ${ }^{14}$ Für ihn ist "Islam und Europa" keine neue Frage.

Lokalisierung ist das Gegenstück zur Globalisierung, ${ }^{15}$ die ein dialektischer Prozess ist: So kann Globalisierung nur auf der Basis

\footnotetext{
9 Beck, U., Globalisierung 44.

10 Saied Reza, A., Glocal Spaces. Globalization and the Power of Religion, in: O'Mahony, A./ Peterburs, W./ Shomali, M.A. (Hg.), Catholics and Shi 'a in Dialogue. Studies in Theology and Spirituality, London 2004, 321-353, unterscheidet daher treffend zwischen ,non-ideological globalization“ und ,ideological (dominated) globalization" (325f.).

11 Es gibt historische Vorstufen, die von verschiedenen Globalisierungstheoretikern unterschiedlich gewichtet werden (vgl. Fürtig, H., Muslime 17f.). Von Muslimen wird auch ein eigener Anteil an der Geschichte der Globalisierung gefordert (19). Vgl. dazu auch Karic, E., Land 45.

12 Vgl. Roy, O., Der islamische Weg nach Westen. Globalisierung, Entwurzelung und Radikalisierung, München 2006, 34, der von „Entterritorialisierung“ spricht. 13 Vgl. Ramadan, T., Muslimsein 182f.; T. Ramadan, Muslims 75. 14 Vgl. Karic, E., Islamic Ethics in a Multireligious Society. Bosnian Experiences and Perspectives for Islam in Europe, http://www.akademiers.de/fileadmin/user upload/pdf archive/schmid/IslamicEthicsKaric.pdf S. 2, der von Globalisierung als „our product“" spricht. Dies ist ein erstaunliches Maß an Identifikation, welches darin begründet ist, dass Karic die bosnischen Muslime als Teil des Westens sieht.

15 Vgl. Schreiter, R., Globalisierung 152 verweist auf „das Globale als
} 
lokaler Bindungen und Kulturen entstehen und beinhaltet stets auch lokale Vielfalt. Folglich kommt es zu einer „(neuen) Betonung des Lokalen“"16, die zur Fragmentarisierung der Welt und der Gesellschaften auch innerhalb der Religionsgemeinschaften sowie zum Aufeinandertreffen lokaler Kulturen $^{17}$ und letztlich zur Dezentralisierung führt. Globalisierung bedeutet nicht nur Homogenisierung, sondern auch neue Vielfalt. Diese Vielfalt entsteht mit dadurch, dass lokale Kulturen sich in ihrer Auseinandersetzung mit der Globalisierung selbst verändern und ganz verschiedene Reaktionsmuster zeitigen. ${ }^{18}$

In der Lokalisierung liegt gerade auch für Religionen mit Universalitätsanspruch wie den Islam ein beträchtliches Konfliktpotential, denn: „Die Vielzahl der kleinen Welten [...] erlaubt keine einheitliche islamische Aussage mehr.“19 Auch der Islam unterliegt somit einem Lokalisierungstrend, was besonders in Europa zu beobachten ist. Daraus ergibt sich nicht zuletzt die Frage, ob hier ein lokaler Islam theologisch möglich ist oder bereits faktisch existiert. ${ }^{20}$ So kann z.B. der bosnische Islam, dem Karic entstammt,

unterdrückende und beherrschende Dimension“. Gleichermaßen kann auch das Lokale einengen und begrenzen. Daher ist ein ausgewogenes Verhältnis von beidem sinnvoll. Vgl. auch Schreiter, R., Katholizität 88f.

16Beck, U., Globalisierung 86. An anderer Stelle spricht Beck von einer ,nichttraditionalistische(n) Renaissance des Lokalen“ (87).

17 Vgl. Beck, U., Globalisierung 90. Daher wird auch der Begriff „Glokalisierung“ verwendet.

18 Vgl. Schreiter, R., Katholizität 30f.

19 Schulze, R., Geschichte der islamischen Welt im 20. Jahrhundert, München 2002, 378. Wie sehr es diese idealisierte Einheit jemals im Laufe der islamischen Geschichte gegeben hat, ist eine andere Frage. Vgl. dazu Kalisch, Muhammad, Abgrenzung im islamischen Denken. Theologische und rechtliche Aspekte, in: Schmid, H./ Renz, A./ Sperber, J./ Terzi, D. (Hg.), Identität durch Differenz? Wechselseitige Abgrenzungen in Christentum und Islam (Theologisches Forum Christentum - Islam), Regensburg 2007, 52-65, 53: „Es hat nie einen einheitlichen Islam gegeben. Es gab und gibt stattdessen unterschiedliche theologische Strömungen, die alle für sich in Anspruch nehmen, den wahren Islam zu lehren."

20 Vgl. dazu die Beiträge im Sammelband: Manger, L.(Hg.), Muslim Diversity. Local Islam in Global Contexts, Richmond 1999, die Lokalisierungstendenzen des Islam in verschiedenen Teilen der Welt zum Gegenstand haben. Der lokale Islam in Europa ist zudem durch eine starke Individualisierung islamischer Identität geprägt, 
als ein lokaler Islam betrachtet werden, wobei bereits diese Einschätzung nicht unumstritten ist. ${ }^{21}$ Bevor jedoch weiter auf die Frage des lokalen Islam eingegangen werden kann, ist zunächst der mediale Islam-Diskurs als prägendste Erfahrung für Muslime in Europa im Kontext der Globalisierung zu berücksichtigen.

\section{Ausgangssituation: Der globalisierte Islam-Diskurs}

Die globalisierte Welt ist eine Welt der Medien. So erweist sich auch das Bild des Islam über weite Strecken als ein Produkt der Medien. Kennzeichen der globalisierten Welt ist, „dass von nun an nichts, was sich auf unserem Planeten abspielt, nur ein örtlich begrenzter Vorgang ist ${ }^{\text {‘22 }}$. Kommunikation wird folglich schwieriger, denn „diese verschiedenen Welten sind potentiell an einem Ort präsent ${ }^{\text {‘23 }}$. Ein Beispiel für die globale Verbreitung von Informationen und globale Interaktionen ist der Karikaturenstreit im Frühjahr 2006: Was in Dänemark in einer Zeitung erscheint, bleibt in anderen Teilen der

\footnotetext{
was inzwischen vielfach empirisch untersucht wurde. Vgl. dazu folgende Bände aus der Buchreihe ,global - local Islam“: Nökel, S., Die Töchter der Gastarbeiter und der Islam. Zur Soziologie alltagsweltlicher Anerkennungspolitiken. Eine Fallstudie, Bielefeld 2002; Frese, Hans-Ludwig, Den Islam ausleben. Konzepte authentischer Lebensführung junger türkischer Muslime in der Diaspora, Bielefeld 2002.

21 Vgl. dazu Balić, S., Bosnien: Paradigma eines europäischen Islam, in: ders., Islam für Europa. Neue Perspektiven einer alten Religion, Köln/Weimar/Wien 2001, 178-192, 181: „Der bosnische Islam hat im Umgang mit Europa eine Erfahrung von mehr als 120 Jahren. "Karic betont stärker universale Züge des Islam und spricht nicht vom „bosnischen Islam“. Selbstverständlich kann auch der bosnische Islam nur in Interaktion mit der islamischen Welt verstanden werden und ist selbst eine polyphone Größe, innerhalb derer Karic eine - wenn auch sehr gewichtige - Stimme darstellt (zu verschiedenen Positionen vgl. X. Bougarel, définitions). Bisweilen wird der Terminus „bosnischer Islam“ auch als Abgrenzungsbegriff gegenüber anderen, vermeintlich intoleranten Ausprägungen des Islam in Europa verwendet. Dagegen ist zu betonen, dass es auch in anderen Teilen Europas und der islamischen Welt vergleichbare islamische Aufbrüche und Erfahrungen einer Minderheitensituation gibt. 22 Beck, U., Globalisierung 30. Vgl. dazu auch Institut für Auslandsbeziehungen (Hg.), Medien ohne Grenzen? Glokalisierung, Journalismus, Kulturelle Identität (Deutsch-arabischer Mediendialog), Stuttgart 2005.

23 Beck, U., Globalisierung 132.
} 
Welt nicht verborgen. Die zeitlich verschobene Rezeption der dänischen Karikaturen in der islamischen Welt ist jedoch zugleich eine Quelle für Missverständnisse, denn eine Karikatur bedeutet dort etwas anderes als in der Tradition des europäischen Journalismus.

Vermittelt durch die Medien spielen umgekehrt wiederum Ereignisse der islamischen Welt im öffentlichen Diskurs in Europa eine zentrale Rolle. Kai Hafez hat den Zusammenhang zwischen Auslandsgeschehen und inländischen Diskursen untersucht und spricht von „foreign news at home“"24. Es kommt dabei zu einer „Verbindung aus Bildkonstruktionen der Nah- und Fernwelt ${ }^{‘ 25}$. Im globalisierten Islam-Diskurs werden dabei lokale Ereignisse oft symbolisch aufgeladen: ${ }^{26}$ Es geht in vielen Diskussionen nicht nur um lokale Probleme, sondern gleich um die globale Konfrontation mit dem Islam, welchem ein Anstreben der Weltherrschaft unterstellt wird. Dies erschwert lokale Lösungen, da vielfach externe Ereignisse in die Argumentationen einfließen. Diesem Mechanismus liegt die Annahme zugrunde, die islamische Welt samt der Muslime in Europa sei ein einheitliches Gebilde. Verbunden mit einer selektiven Wahrnehmung, Worst-Case-Denken und einer Dekontextualisierung entsteht dann ein Feinbild-Diskurs mit dem Islam als Gegenbild. ${ }^{27}$ In diesem Diskurs erfahren sich viele Muslime als Objekte, die der westlichen Welt und ihren Medien machtlos gegenüber stehen. Muslime sehen sich folglich häufig in der Position der Angeklagten, in der Rolle derer, die sich für Ereignisse wie Selbstmordattentate zu entschuldigen haben, mit denen sie nichts zu tun haben. ${ }^{28}$ Natürlich

24 Hafez, K., Die politische Dimension der Auslandsberichterstattung, 2 Bde., Baden-Baden 2002, Bd. 2, 162f., 265, 310.

25 Hafez, K., Dimension, Bd. 1, 176.

26 Ein Beispiel dafür ist auch der Bosnienkrieg, vgl. dazu Akbar, S. A./ Donnan, H., Islam in the Age of Postmodernity, in: ders. (Hg.), Islam, Globalization and Postmodernity, London/New York 1994, 1-20, $7 \mathrm{f}$.

27 Vgl. Hafez, K., Dimension, Bd. 2, 228, 301, 231f. Vgl. auch Schmid, H., Ein schwieriges Verhältnis. Muslime und Öffentlichkeit in Deutschland, in: HerKorr 60 (2006), 75-79, 75-77.

28 Vgl. Ayish, M., World 89. 
wäre auch zu diskutieren, ob diese Opferrolle immer berechtigt ist und wo sie möglicherweise klar instrumentalisiert wird. ${ }^{29}$

Ausgangspunkt nicht zuletzt auch für die theologische Reflexion der Muslime in Europa sind daher in erster Linie negative Erfahrungen mit den Medien und dem in ihnen geführten Islam-Diskurs. ${ }^{30}$ In einer solchen Situation liegen positive Deutungen von Globalisierung zunächst nicht nahe. Interessanterweise wird trotz aller sonstigen Unterschiedlichkeit der Ansätze sowohl von Karic als auch von Ramadan die Rolle der Medien klar negativ gesehen. Karic spricht plakativ von ,the antimuslim hysteria of the media demiurge ${ }^{631}$ und betrachtet das Islambild der Medien als Fortsetzung von Fehlwahrnehmungen in der Geschichte. Ramadan sieht die Diskriminierung von Muslimen in Europa als unmittelbare Folge des von außenpolitischen Ereignissen bestimmten medialen Islambildes. ${ }^{32}$ In diesem Punkt besteht ein breiter muslimischer Konsens. Es handelt sich um eine sehr prägende Erfahrung, die möglicherweise zu einer globalen muslimischen Solidarisierung führt.

Ein weiterer Aspekt kommt hinzu: Medien und Internet ermöglichen den eingewanderten Muslimen in Europa weiterhin den Zugang zum Diskurs und zur Kultur der Herkunftsländer. Dies erschwert letztlich eine Lokalisierung bzw. Kontextualisierung, so dass der Islam in vielen westeuropäischen Staaten noch weitgehend von Außeneinflüssen geprägt ist. ${ }^{33}$ Über Satellitenfernsehen und andere

29 Vgl. dazu Malik, J., Nationale und religiöse Selbst- und Fremdbilder der Muslime in Deutschland, in: Haupt, H-G./ Langewiesche, D. (Hg.), Nation und Religion in Europa: Mehrkonfessionelle Gesellschaften im 19. und 20. Jahrhundert, Frankfurt 2004, 283-302, 302; R. Ghadban, Ramadan 162.

30 Vgl. Akbar, S. / Donnan, H., Islam 9f.

31 Karic, E. Land 110ff. Vgl. auch 20, 51.

32 Vgl. Ramadan, T., Muslimsein 243. Vgl. auch 268; Ramadan, T., The Global Ideology of Fear or the Globalization of the Israel Syndrome (8 December 2005), in: http://www.tariqramadan.com, 4.

33 Vgl. Roy, O., Weg 139 sowie die entsprechende Übersicht zum Islam türkischer Herkunft in Deutschland bei Lemmen, Thomas, Islamische Vereine und Verbände in Deutschland, Bonn 2002, 104, die deutlich macht, wie sehr sich in den islamischen Organisationen in Deutschland der türkische Diskurs abbildet. 
Medien erreicht die Muslime aus den Herkunftsländern möglicherweise auch ein stärker negatives Bild der Globalisierung.

Die besondere Situation der Muslime in Europa, die letztlich im Höchstmaß mit Globalisierung und deren Folgen konfrontiert sind, besteht darin, dass sie von der islamischen Welt als Randphänomen betrachtet und in Europa selbst ausgegrenzt werden. Man kann diese Situation mit Ramadan als einen ,double inferiority complex “34 bezeichnen. Zusätzlich zum als äußerst negativ wahrgenommenen Mediendiskurs sind die europäischen Muslime spezifisch mit einer Europa-Diskussion konfrontiert, oft dergestalt, dass der Islam als fremd definiert wird. So sind auch die beiden hier zu untersuchenden Autoren von Ausgrenzungserfahrungen gezeichnet: Ramadan teilt die Diskriminierungserfahrung eingewanderter Muslime und hält in der Konsequenz beiderseitige Schritte der Öffnung für notwendig. ${ }^{35}$ Karic erfährt, wie Bosnien mit seinem autochthonen Islam von Europa als Randgebiet behandelt wird. Daher betont er den Islam als einen Pfeiler Europas und besteht auf dessen Zugehörigkeit: „Islam forms part of the foundation of Europe, as does the Bibel and ancient Greece. ${ }^{\text {‘36 }}$

\section{Organisationsformen: Netzwerkbildung und Institutionalisierung im ,europäischen Islam“6}

Die Globalisierung wirkt sich auch auf die Identität der Muslime und ihre Organisationsformen aus. ${ }^{37}$ So wie der Einwanderungsprozess

34 Ramadan, T., Muslims 225.

35 Vgl. Ramadan, T., Muslimsein 268, 187ff. (thematisiert zunächst die

Zugehörigkeit zur islamischen Umma), 232ff.

36 Karic, E., Land 65. Vgl. auch 38 und Karic, E., Bosnia 78ff. Kennzeichnend für

Karics Sicht ist schon der Buchtitel „Never-Never Land“, der auf die fiktive Insel

Peter Pans „Neverland“ (manchmal auch „Never Never Land“ oder „Never Never Never Land") anspielt und symbolisiert, wie weit entfernt Karic Bosnien von Europa sieht.

37 Vgl. Schreiter, R., Kontextualität 40: „Nach dem Angriff der Globalisierung müssen die Identitäten neu gestaltet werden." 
nach Europa ungeplant verlief und einen ungeplanten Daueraufenthalt im Einwanderungsland nach sich zog, verlief auch die Institutionalisierung der Muslime zunächst ungeplant. Sie wurde nur teilweise von theologischen Konzepten begleitet, so dass auch hier zunächst die Frage der Organisationsformen zu überblicken ist, bevor dann theologische Konzepte vorgestellt und diskutiert werden.

Globalisierung bedeutet stets auch eine innere Pluralisierung, die Ungleichzeitigkeiten mit sich bringt. $\mathrm{Da}$ der Islam keine Zentralinstanz wie die katholische Kirche kennt, ${ }^{38}$ fällt ein zentraler institutioneller Lokalisierungswiderstand weitgehend aus. Die entscheidende Bezugsgröße im Islam ist wie im Judentum lokal. Somit kann man den Islam als eine globale Idee mit lokalen Verwirklichungsformen bezeichnen. ${ }^{39}$ Institutionalisierung im Einwanderungsland wächst dementsprechend auch von unten nach oben und gelingt zunächst besser auf lokaler Ebene. ${ }^{40}$

Die klassischen Verbände z.B. in Deutschland sind jedoch oft überfordert im Interessenkonflikt zwischen Herkunftsland- und Deutschland-Orientierung. Trotz Einflussnahme aus den Herkunftsländern und dem Festhalten an den Herkunftssprachen als „Moscheesprachen“ bildet sich unter den Muslimen Europas eine Vielzahl lokaler Identitäten heraus. Die lokale Diversifizierung reicht bis hin zur Unüberschaubarkeit. Nicht zuletzt die Integration in das lokale Bildungssystem durch Einführung von Islamischem Religionsunterricht schafft einen neuen lokalen Islam, der sich vom Islam der Koranschulen unterscheidet. ${ }^{41}$ Es entsteht ein „europäischer

38 Schreiter, R., Katholizität 33f. schätzt dieses auch zurückhaltend ein: „Die römisch-katholische Kirche mag unter Johannes Paul II. anstreben, ein globales System zu werden, kann aber Kontrolle nicht auf allen Ebenen erreichen [...].“ 39 Die Strömung des Panislamismus stellte den Versuch einer islamischen Einigung als Antwort auf die Erfahrungen des Kolonialismus dar. Möglicherweise führt die Globalisierung zu einer ähnlichen Reaktion. Vgl. auch Schulze, R., Islamischer Internationalismus im 20. Jahrhundert. Untersuchungen zur Geschichte der Islamischen Weltliga, Leiden 1990; Grundmann, J., Islamische Internationalisten, Strukturen und Aktivitäten der Muslimbruderschaft und der Islamischen Weltliga, Wiesbaden 2006.

40 Vgl. Lemmen, Th., Vereine 25-27.

41 Vgl. Schmid, H., Neue Phase des Zusammenlebens. Schritte auf dem Weg 
Islam“, auch wenn sich Muslime diesem Begriff häufig widersetzen. ${ }^{42}$ Viel zuwenig wird gesehen, dass lokale Gemeinden als Ergebnisse von bottom-up-Entwicklungen sich oft stark von dem dem Islam als typisch Zugeschriebenen unterscheiden. So sind muslimische Vereinigungen in Europa „keine bloße Fortsetzung alter Formen, da auf die Bedingungen der Migrationssituation im Aufnahmeland reagiert wird“43.

Globalisierung und Lokalisierung verbindende Organisationsformen der Zukunft sind möglicherweise internationale Netzwerke: „Das Netz bildet einen unbestimmten Raum, in dem sich die Muslime, die die eine oder andere Ausprägung des Islam teilen, sammeln können $[\ldots] .{ }^{* 44}$ Besonders wirksam ist die Bewegung Fethullah Gülens, deren Netzwerkstruktur erst den Erfolg der Bewegung ermöglicht. Lokale Teilnetzwerke sorgen in der Bewegung dafür, dass Diskurse lokal angepasst werden können. ${ }^{45}$

Auch die beiden hier behandelten Autoren nehmen auf die Frage der Organisationsform Bezug: Ramadan wünscht sich Organisationen, die

zum Islamischen Religionsunterricht, in: HerKorr 59 (2005) 239-244; Behr, H., Curriculum Islamunterricht. Analyse von Lehrplanentwürfen für islamischen Religionsunterricht in der Grundschule. Ein Beitrag zur Lehrplantheorie des Islamunterrichts im Kontext der praxeologischen Dimension islamisch-theologischen Denkens, diss. phil., Bayreuth 2005, 229-457.

42 Karic spricht unbefangen von „Euro-Islam“ (E. Karic, Land 11-29), während Ramadan von „Musulman Européen“ spricht und der Begriff „Euro-Islam“ lediglich im Vorwort von Richard Friedli vorkommt (vgl. Muslimsein 11f.). - Hierbei ist eine klare Differenzierung wichtig: Bochinger, Christoph, Islamunterricht in Deutschland aus religionswissenschaftlicher Sicht, in: Bauer, Thomas u.a. (Hg.), Islamischer Religionsunterricht. Hintergründe, Probleme, Perspektiven, Münster 2004, 21-28, 22f. verdeutlicht, dass es sich bei „Euro-Islam“ zunächst um keine Selbst-, sondern um eine Außenwahrnehmung handelt, was beides klar voneinander zu trennen ist. Insbesondere kann die Außenwahrnehmung nicht als normativ für die Selbstdefinition gelten, aber sie kann wiederum Rückwirkungen auf die Selbstdefinition haben. 43 Hadeed, A., Selbstorganisation im Einwanderungsland. Partizipationspotentiale von Migranten-Selbstorganisationen in Niedersachen, Oldenburg 2005, 235.

44 Allam, F., Der Islam in einer globalen Welt, Berlin 2004, 163.

45 Vgl. Agai, B., Zwischen Netzwerk und Diskurs. Das Bildungsnetzwerk um Fethullah Gülen (geb. 1938), Schenefeld 2004, 364, sowie die Homepage der Bewegung http://de.fgulen.com/. 
finanziell unabhängig und frei von ausländischem Einfluss zunächst auf regionaler Ebene tätig sind. ${ }^{46}$ Ramadan sieht verschiedene Stufen der Organisationen: zunächst ethnisch, dann islamisch für verschiedene soziale Belange, schließlich eine noch anzustrebende dritte Stufe: nicht nur muslimisch, auf der Basis gemeinsamer Werte für gemeinsame Projekte. Er propagiert den Slogan ,commit yourself with the Other for dignity, justice, and peace, for the Other as well as for yourself ${ }^{\text {s } 47}$. Dieser Ansatz ist handlungsorientiert. Im gemeinsamen Engagement gegen Globalisierung sieht Ramadan einen Motor für die Organisationsbildung. Dabei bleibt jedoch offen, wie die Vermittlung nichtislamischer und islamischer Werte und Prinzipien als Basis für solche Organisationen gelingen kann. ${ }^{48}$

Karic sieht den Weg der Muslime nur in Europa und steht daher jeglichem Einfluss von außerhalb kritisch gegenüber. Er geht vom bosnischen Modell eines institutionalisierten Islam aus. ${ }^{49}$ Bei anderen Exponenten des bosnischen Islam steht die Frage der Institutionalisierung allerdings stärker im Vordergrund, so bei Mustafa Cerić, der das bosnische Organisationsmodell für ganz Europa propagiert. $^{50}$ Dass Karic weniger Gewicht auf die Frage der Organisationsform legt, zeigt, dass er mehr Denker als Aktivist ist, was im nächsten Teil Berücksichtigung finden wird.

46 Ramadan, T., Muslimsein 273-275.

47 Ramadan, T., Muslims 158. Vgl. auch 157 sowie Ramadan, Tariq, Interreligious Dialogue from an Islamic Perspective, in: Timmerman, C./ Segaert, B.(Hg.), How to Conquer the Barriers to Intercultural Dialogue. Christianity, Islam and Judaism, Brüssel u.a. 2005, 85-100, 98-100.

48 Siehe dazu unten 5.3. Möglicherweise handelt es sich hier um eine der Spannungen im Gedankengebäude Ramadans.

49 Vgl. zu diesem Modell Karcic, F., Bosniaks. Trotz externer Versuche der Einflussnahme konnten auch nach dem Krieg die bosnischen Muslime ihre lokale Identität bewahren (so auch die Einschätzung bei Bremer, Thomas, Kleine Geschichte der Religionen in Jugoslawien. Königreich - Kommunismus - Krieg, Freiburg 2003, 105). Bei Karic, E., Bosnia 235, 239 ist eine vom Staat getrennte Institutionalisierung des Islam angedeutet.

50 Vgl. Kinzler, J., Schwieriges Zusammenleben. Bosnien-Herzegowina zehn Jahre nach Kriegsende, in: HerKorr 59 (2005) 423-428, 425. 


\section{Theologische Modelle: Islam als Gegner oder Reformkraft der Globalisierung?}

\subsection{Konkurrierende Universalitätsansprüche}

Die mit der Globalisierung verbundene theologische Fragestellung hängt besonders damit zusammen, dass der Islam eine Religion mit Universalitätsanspruch ist. ${ }^{51}$ Dieser Anspruch liegt in der Botschaft des Koran begründet. Dort heißt es z.B.: „Ich bin der Gesandte Gottes an euch alle.“ (Sure 7,158) Oder: „Und wir haben dich gesandt, damit $\mathrm{du}$ den Menschen allesamt ein Verkünder froher Botschaft und eine Warner seiest." (Sure 34,28, vgl. auch 6,90) Angesichts der Globalisierung ist der Islam mit diesem Anspruch doppelt herausgefordert:

Zum einen im unmittelbaren Verhältnis zur Globalisierung, die auch einen insbesondere durch Befürworter des Wirtschaftsliberalismus propagierten ethischen Rahmen mit universalen Werten umfasst und traditionelle Werte teilweise in Frage stellt: ${ }^{52}$ Globalisierung stellt die Religionen und damit auch den Islam (ähnlich wie in der Konfrontation mit Moderne) letztlich vor die Entscheidung, ob sie sich annähern und einbringen wollen oder ein Gegenmodell entwerfen. Dies mündet in die Frage, ob die Globalisierung als Konkurrenz oder Chance gesehen wird.

Zum anderen im Umgang mit der partiellen Realisierung des eigenen Anspruchs, denn in der Epoche der Globalisierung wird deutlicher denn je erfahrbar, dass es andere Religionen gibt. Auch hier sind wiederum zwei Reaktionsmuster denkbar: verstärkte Mission bzw. $d a ' w a$, wie durch nach dem Ende des Kalifats bezüglich Trägern und

$51 \mathrm{Vgl}$. Zirker, H., Christentum und Islam. Theologische Verwandtschaft und Konkurrenz, Düsseldorf 21992, 78f.

52 Schreiter, R., Globalisierung 149, nennt folgende Werte, die jedoch nur für einen

Teil der Menschen Wirklichkeit geworden sind: „Fortschritt, Einbeziehung in die

Nutznießung der Globalisierung und Gleichberechtigung als produzierendes und konsumierendes Subjekt". 
Arbeitsgebieten universalisierte muslimische $d a$ 'wa-Bewegungen geschehen, ${ }^{53}$ oder Dialog und Akzeptanz der anderen Religionen. ${ }^{54}$

Bei beiden Fragen geht es nicht um nur zwei Alternativen, sondern um zwei Pole, zwischen denen sich wie auf einer Skala verschiedene Positionen anordnen. Jetzt sind die Werke der beiden Autoren daraufhin zu untersuchen, wobei deren "Globalisierungstheologien“" zunächst kurz zu umreißen sind. Dabei wird von deren Bewertungen der Globalisierung ausgegangen (a). Es folgt eine Darstellung der hermeneutischen Grundlagen (b). Schließlich geht es um die Perspektiven für europäische Muslime zwischen lokalen Erfahrungsräumen und globalen Werten aus der Sicht des jeweiligen Autors (c).

\subsection{Enes Karic: „universal Islam“ als Beitrag zu einer ganzheitlichen Globalisierung}

a) Karic blickt wie folgt auf die Globalisierung: „We are faced today with the huge question whether present-day globalization will take place with the mediation of modern scientistic scholarship, that knows nothing either of God or of dialogue, or whether the spiritual component from the treasury of the traditional teachings of the great world religions - Judaism, Christianity, Islam, Buddhism ... contribute to these current processes." ${ }^{, 55}$ Karics Sicht der Globalisierung ist somit ambivalent: Globalisierung kann sich negativ auswirken als atheistische und materialistische Herrschaftsglobalisierung analog zum Kolonialismus, womit sie eine „westernization in disguise“ ${ }^{\text {“56 }}$ wäre. Globalisierung kann aber auch eine positive Entwicklung sein, sofern sie ganzheitlich ist und vom Beitrag der Religionen profitiert, wobei es Karic um alle großen

53 Vgl. Wrogemann, H., Islam 185f., $189 f$.

54Allerdings müssen da'wa und Dialog kein Widerspruch sein. Vgl. Wrogemann, H., Islam 414-417; Schmid, H., Wozu Dialog? Zielbestimmungen und ihre Problematik im Kontext der aktuellen christlich-islamischen Beziehungen, in: Hilberath, Bernd Jochen/ Kuschel, K-J. (Hg.), Theologie im Gespräch. Eine Agenda für die Zukunft. FS Urs Baumann, Frankfurt 2006, 171-190.

55 Karic, E., Land 34. Hervorhebungen im Zitat von H.S.

56 Karic, E., Land 47. Vgl. dazu auch 44-48. 
Religionen gleichermaßen geht. ${ }^{57}$ Es ist eine offene Frage, ob die positiven oder negativen Züge überwiegen, so dass kein Rückzug der Religion, sondern im Gegenteil der mit dem Verb to contribute umschriebene aktive Einsatz für eine adäquate Globalisierung geboten ist, welche dann als ,islamically inspired globalization“58 ${ }^{\text {(58 }}$ gelten kann. Diese immer partielle Mitgestaltung vollzieht sich auf der Basis der vorauszusetzenden Säkularisierung und ist nicht im Sinne einer Islamisierung $\mathrm{zu}$ verstehen. Aus der Sicht des Islam ist also in mehrfacher Hinsicht ein Dialog erforderlich: einerseits mit den anderen Religionen, andererseits mit den anderen Wissenschaften und gesellschaftlichen Kräften.

b) Grundlage für eine positive Sicht der Globalisierung ist die Universalität und damit unerschöpfliche Deutungsoffenheit ${ }^{59}$ des Korans, welche seine Kommunikationsfähigkeit ermöglicht und die Relevanz seiner Botschaft heute garantiert: „If the Qur'ān is a universal book, the Muslims must deal universally not only with the Qur'ān but also with the world, this world, despite all the difficulties they meet. ${ }^{\text {" }}{ }^{60}$ Der aufgeschlossene Umgang mit Globalisierung wird aus dem Koran begründet; die Globalisierung und der Koran sind beide universal ebenso wie Gott, der kein islamischer Gott ist. ${ }^{61}$ Karic sieht zugleich sein hermeneutisches Verständnis des global zu verstehenden Koran als Gegenmodell zu fundamentalistischen wie zu rein modernistischen Ansätzen. Aufgrund der Universalität des Korans steht an erster Stelle die gesamte Menschheit. Es kommt zu keiner Aufteilung in Gläubige und Ungläubige; alle Erfahrungen werden in dieser Weltsicht als gleichwertig erachtet. Wie die Muslime zur Globalisierung beitragen, so profitieren sie auch von den

57 Vgl. Karic, E., Land 31, 34f., 45, 95. Zur Globalisierung als Chance vgl. E. Karic, Ethics 2.

58 Karic, E., Land 37. An anderer Stelle spricht er von einem ,islamically acceptable globalization project“ (43). Dass Karic Globalisierung in einem umfassenden, nicht auf aktuelle Entwicklungen beschränkten Sinn sieht, zeigt sich an seiner Formulierung „present-day globalization“ im obigen Zitat.

$59 \mathrm{Vgl}$. Karic, E., Bosnia 243. So spricht Karic auch vom ,need to re-read the Qur'ān" (180).

60Karic, E., Bosnia 181.

$61 \mathrm{Vgl}$. Karic, E., Land 40f. 
Erfahrungen anderer Menschen und Religionen, die bemerkenswerterweise wiederum in die Interpretation des Korans einfließen: „Just as the Qur'ān was not revealed to the Muslims alone, but to humanity as a whole, thus Muslims, in the interpretation of the Qur'ān, must take into account and use the experience of interhumanity, not merely the experience of the Muslim world."62 Damit ist ein hermeneutisches Grundprinzip für interkulturellen Dialog aus islamischer Perspektive entwickelt.

c) Schließlich sieht Karic in der Globalisierung einen Impuls zur Reform und Selbstuniversalisierung des Islam. Oft werden lokale Traditionen wie Bekleidungsgewohnheiten zu universalen religiösen Identitätsmarkern erhoben, so dass es $\mathrm{zu}$,clashes between the universal and the local ${ }^{\text {"63 }}$ kommt. Karic steht kritisch $\mathrm{zu}$ einer Lokalisierung, die Lokales mit einem universalen Anspruch versieht. Er propagiert daher einen universalen Islam als offene und interpretationsbedürftige Größe, die kommunikationsfähig mit anderen Religionen ist und in erster Linie ethische Prinzipien umfasst: „One of the ways that European Muslims could gain an Islamic West in the field of their culture is through the universalization of their Islamic life and thought. Today's European Muslims are faced with that important task of self-universalization, since it is not possible to be a good Muslim nor a successful European by remaining merely at the local level, the level of the homeland. [...] Universal Islam is for all of us, and local Islamic customs should be left to the home, the family, to the national culture. “64 Die Schaffung eines „Islamic West“" geschieht nicht auf der politischen, sondern auf der kulturellen Ebene, geht es doch darum, islamische und europäische Identität miteinander $\mathrm{zu}$ verbinden und nicht als Gegensatz zu betrachten. Ziel für Karic ist schließlich ein universaler Euro-Islam: „Euro-Islam“ would be a universally interpreted Islam, which would liberate European Muslims from their self-ghettoization and from the ghettoization of Islam in Western Europe."“65

62 Karic, E., Bosnia 234.

63 Karic, E., Land 66. Vgl. dazu auch Karic, E., Ethics 4.

64 Karic, E., Land 66.

65 Karic, E., Land 28. 


\subsection{Tariq Ramadan: „muslim resistances“ und die islamische Alternative}

a) Ramadans Position zur Globalisierung ist in folgendem Zitat kompakt zusammengefasst: „The globalization with which we are presented and that is imposed upon us today sanctions above all the absolute primacy of the logic of economics over every other consideration, and the efficiency of communication networks and highways seems to draw us more and more into becoming better consumers. The picture would be very dark were it not for a widespread movement of resistance: when faced with neoliberal economics, the message of Islam offers no way out but resistance. In the West, as in the East, we are summoned to use our minds, our imaginations, and our creative abilities to think of an alternative using our sources in partnership with all those who resist and mobilize for ,alternative ways" "66 Bei Ramadan steht eindeutig die Globalisierungskritik im Mittelpunkt. Er sieht Globalisierung in erster Linie als eine ökonomische Ideologie und Macht, auf die auch andere Aspekte der Globalisierung wie Kommunikationstechnologie hingeordnet sind und von der er ein dunkles Bild zeichnet. Daran schließt sich eine „erhellende“ Antithese an, die er als einzigen Ausweg aus der bedrohlichen Lage kennzeichnet: Reaktion kann nur eine „muslim resistance“ sein, denn die islamischen Prinzipien stehen in einem deutlichen Gegensatz zur Ideologie der Globalisierung. ${ }^{67}$ Konsequenz aus der entschiedenen Globalisierungskritik ist, dass Ramadan von der Notwendigkeit von Alternativen (,alternative ways $\left.^{6}\right)^{68}$ spricht. Zunächst sind die islamischen Quellen (,our

66 Ramadan, T., Muslims 173. Hervorhebungen im Zitat von H.S.

67 Vgl. besonders die fünfsprachige Publikation: Ramadan, Tariq, Globalisation. Muslim Resistances, Lyon 2003. Ein Buchkapitel mit dem Titel „economic resistance" findet sich bei Ramadan, T., Muslims 174-199. Die Negativbewertung des neoliberalen Wirtschaftssystems reicht soweit, dass Ramadan es als dār al-harb bezeichnet (176). Im französischen Original ist von „résistance“ die Rede, was auch die Bezeichnung für die Widerstandsbewegung in Frankreich gegen den Nationalsozialismus ist. Es handelt sich also um eine in der Tat sehr deutliche Positionierung.

68 Vgl. Ramadan, T., Muslims 173, 175, 191, 198 u.ö. Vgl. auch Hofmann, M., Der Islam als Alternative, München 1992. 
sources") dafür zu verwenden. Ramadan sieht sich jedoch auch in einem Boot mit anderen Globalisierungskritikern, mit denen Muslime gemeinsam Widerstand leisten könnten. Er kritisiert allerdings an anderer Stelle, dass die Globalisierungsgegner eurozentristisch ausgerichtet sind und Muslime nicht beteiligen, wogegen er betont, „dass die globalisierungskritische Bewegung, die schließlich eine andere Globalisierung erstrebt, nur dann eine Zukunft haben wird, wenn sie in einem offenen und fruchtbaren Dialog mit der Welt des Islam eintritt. “69

b) Die vorgeschlagene Alternative zur Globalisierung beruht auf den universalen islamischen Prinzipien. Die Globalisierung erfordert für Ramadan eine universale Antwort, die er als „more global“ kennzeichnet: „We have here suggested an alternative way of looking at things that is more global and based on the nature of the actual situation and fields of activity and their relation to the universal principles and ethic of Islam.“" ${ }^{40}$ Und: „Das globale Denken, dessen wir so dringend bedürfen, verlangt, dass wir uns auf unsere universellen Perspektiven besinnen und deren grundlegende Ziele und Zwecke erfassen." "71 Mit diesem universalen Ansatz setzt sich Ramadan klar von lokalen ethnischen Traditionen ab. ${ }^{72}$ Dabei propagiert er eine Rückkehr zu den Quellen, die einer zeitgemäßen Relecture bedürfen. Die gegenwärtige Situation wird dabei in Beziehung zu den universalen Prinzipien gebracht; im Blick auf die Wirtschaftsordnung kommt der Almosensteuer und dem Zinsverbot

69 Vgl. Ramadan, T., Armut 3. Vgl. ders., Muslims 199. Beim Weltsozialforum im November 2003 in Paris hielt er einen Vortrag (vgl. O. Roy, Weg 328f.; Eussner, Gudrun, ATTAC - At-Taqiya - Attacke. Der Islamist Tariq Ramadan und die Globalisierungskritiker vereint im Kampf, http://www.eussner.net/artikel 2004-0316_00-22-23.html). Zu den engen Beziehungen zu Globalisierungskritikern vgl. R. Ghadban 64, 67. Eine mit Ramadan durchaus vergleichbare globalisierungskritische Stimme in Deutschland ist die „Islamische Zeitung“ (vgl. dazu Schmid, H., Verhältnis 78).

70 Ramadan, T., Muslims 195. Vgl. 191, 195.

71 Ramadan, T., Globalisation 96. Vgl. auch T. Ramadan, Muslims 177 und T. Ramadan, Ideology 5. Zur Methode Ramadans vgl. Ghadban, R., Ramadan 38 und McAuliffe, J. D., Reading the Qur'ān with Fidelity and Freedom, in: JAAR 73 (2005), 615-635, 627f.

72 Vgl. Ramadan, T., Muslims $215 f$. 
besondere Bedeutung zu. ${ }^{73}$ Es fällt gleichwohl auf, dass jetzt die Perspektive eine rein islamische ist und Ramadan folglich in seinen Werken fast ausschließlich islamische Quellen zitiert.

c) Globalisierung erfordert Wandel und Anpassung an das Globale. ${ }^{74}$ Daraus ergeben sich in der Folge verschiedene Möglichkeiten: Einerseits die einer lokalen Identität in Abgrenzung nach dem Motto „,in Europa außerhalb Europas leben“ oder aufgrund äußeren Anpassungsdrucks als „europäische Muslime ohne Islam“75. Beides scheidet für Ramadan aus. Seine Vision ist die einer lokalen Identität im Austausch; diese umfasst „Präsenz, Austausch, Teilnahme und Geben: das ist sehr viel mehr, als bloß integriert zu werden“"76. „Eine Zukunft für die Muslime in Europa lässt sich nicht vorstellen, wenn sie sich weigern müssen, Beziehungen mit ihrer Umgebung aufzunehmen, und wenn sie keine dialektische Beziehung entwickeln, dank derer sie sein, geben und von dem anderen empfangen können. Die Gesetze erlauben in einem weiten Maße eine geachtete Präsenz; und die Lehren des Islam gebieten den Muslimen, aus jeder Sache, aus jeder Gelegenheit Nutzen $\mathrm{zu}$ ziehen, insofern dies nicht in Widerspruch zu einem Prinzip ihrer Religion steht. “" ${ }^{\text {"77 }}$ Eine Perspektive für die europäischen Muslime besteht somit in einer „dialektischen Beziehung“" zu ihrer Umgebung, wobei die islamischen Prinzipien letztlich die oberste Instanz darstellen. Eine gewisse Distanz gegenüber den westlichen Gesetzen zeigt sich auch in der Formulierung ,in einem weiten Maße“. Auch wenn sich Ramadan für eine islamische Öffnung ausspricht, sind somit klare Grenzen in den Beziehungen mit der nichtislamischen Umgebung gesetzt.

\subsection{Zweierlei Universalismus - Karic und Ramadan im Vergleich}

Beide Ansätze sind Exponenten einer Theologie für den europäischen Kontext. Während Karic als ein kritischer Globalisierungsbefürworter

73 Vgl. Ramadan, T., Muslims 22.

74 Vgl. Ramadan, T., Muslimsein 227.

75 Ramadan, T., Muslimsein 229 bzw. 224.

76 Ramadan, T., Muslimsein 233.

77 Ramadan, T., Muslimsein 265f. Dass Ramadan die Säkularisierung kritisch sieht, zeigt sich dort 261, 267, 276, 279. 
gelten kann, der sich dezidiert vom negativen Globalisierungverständnis der islamischen Welt absetzt, ${ }^{78}$ ist in Ramadan eher ein Exponent muslimischer Gegenglobalisierung ${ }^{79} \mathrm{zu}$ sehen, die naheliegenderweise auf islamische Prinzipien zurückgreift. Karic teilt zwar einige Argumente von Globalisierungskritikern, wenn er den Materialismus und seelenlosen Konsum anprangert. ${ }^{80}$ Bei Ramadan ist die Antithese jedoch prinzipiell, woraus letztlich eine Spannung zu dem von ihm vertretenen Konzept einer dialektischen Beziehung mit der Umgebung entsteht. ${ }^{81}$ Ramadan bezieht sich allein auf Globalisierung als neoliberale Ideologie, wodurch mögliche positive Errungenschaften aus dem Blick geraten. ${ }^{82}$

Als Ausgangspunkt stand die doppelte Frage nach der Position zur Globalisierung und der zu anderen Religionen (siehe 5.1.). Bei Karic lässt sich beobachten, dass eine Öffnung in beiden Fragen miteinander einhergeht, während bei Ramadan mit der Globalisierungsopposition zumindest eine Skepsis und relative Zurückhaltung im interreligiösen Dialog korreliert ${ }^{83}$ und für ihn die Selbstbehauptung des Islam wichtiger als eine Öffnung ist. Die Unterschiedlichkeit beider Positionen ergibt sich letztlich aus der theologischen Hermeneutik von Ramadan und Karic: Beide Autoren sind wesentlich vom Koran inspiriert. Karic arbeitet jedoch mit einer modernen semiotischen

78Vgl. Karic, E., Land 47: „This gives a bad image to globalization, so that globalization is often regarded in the Islamic world, rightly or wrongly, in the same way as modernization, as westernization in disguise."

79 Vgl. zu dieser Kategorie Fürtig, H., Muslime 45; Riddell, P. G., Christians, 103; Renz, A., Globalisierung 97-100.

$80 \mathrm{Vgl}$. Karic, E., Land 44.

81 Diese Spannung entsteht möglicherweise dadurch, dass Ramadan eine scharfe Globalisierungskritik und ein Modell zur „Integration“ der Muslime in Europa miteinander kombiniert. Hierin kann eine der von Ghadban herausgearbeiteten Mehrdeutigkeiten des Diskurses Ramadans gesehen werden (vgl. Ghadban, R., Ramadan 36f., 67f. u.ö.).

82 Siehe dazu oben Anm. 10.

83 So auch die Einschätzung bei Valkenberg, T., Sharing 326. Ramadan votiert vor allem für einen Dialog des Handelns und spricht davon, dass „ein authentischer Dialog [...] nur auf der Grundlage einer großen gemeinsamen Anstrengung des Widerstands gegen den menschlichen Wahnsinn, Ungerechtigkeit und Ausbeutung“ (Ramadan, T., Globalisation 103) möglich ist. Vgl. dazu auch Zemouri, A., Ramadan 127, wo Ramadan besonders auf seine Dialogkontakte verweist. 
Hermeneutik. Ramadan schwankt zwischen einer wörtlichen und einer kritisch-kontextuellen Lesart, auch wenn er teilweise die Vielfalt an möglichen Deutungen herausstellt. ${ }^{84}$

Beide Autoren positionieren sich in der oben beschriebenen Dynamik zwischen Globalisierung und Lokalisierung auf Seite des Globalen, nicht jedoch im Sinne der klassischen Globalisierungsideologie. Ramadan vertritt eine ,alternative Globalisierung“. Karic propagiert letztlich eine Reform der Globalisierung. Beide halten damit an der Idee des Universellen fest und setzen der Globalisierung nicht etwa eine stärkere Betonung des Lokalen entgegen. ${ }^{85}$ Ramadans Grundlage dafür ist die Universalität islamischer Prinzipien, die er mit gegenwärtigen Ansprüchen vermittelt und der Globalisierungsideologie kritisch entgegen stellt. Karic sieht die verschiedenen Religionen auf einer Ebene und hebt deren Kommunikationsfähigkeit und gemeinsames Potential hervor. Karics Entwurf trägt dem universalen Anspruch des Islams und dessen nur partikularer Verwirklichung Rechnung und ist von einer hohen Lernbereitschaft gegenüber verschiedenen Kulturen gekennzeichnet. ${ }^{86}$ Er verabsolutiert nicht die eigene Kultur, sondern trennt Glaube und kulturelle Identität, indem „Religion zu einem universellen Kodex von Normen erhoben wird “87. Universalität bedeutet für Karic Übersetzbarkeit des Islam in verschiedene Kontexte. So hat Universalität für Karic schließlich auch eine politische Komponente: Ebenso wie ,arabisch“ und ,islamisch“ nicht identisch sind, sind auch „europäisch“ und „,christlich“ nicht identisch ${ }^{88}$ Die Universalität der Religionen steht damit kulturalistischen Konzepten der Ausgrenzung

84 Vgl. Ramadan, T., Barriers 91, 95. Dazu kritisch Ghadban, R., Ramadan 131. 85 Dies entspricht auch der grundsätzlichen Einschätzung bei P. G. Riddell, Christians 103: „Muslim criticism of western-driven globalization does not advocate its replacement by greater regionalism and localization."

86 Anders bei Ramadan, der höchstens assoziative Bezüge zum Christentum und zur Philosophie herstellt (vgl. dazu auch kritisch Ghadban, R., Ramadan 17). Selbst beim Dialog argumentiert Ramadan allein aus islamischen Quellen. Vgl. den Grundsatz bei T. Ramadan, Muslims 68 ,we must go back to the sources of Islamic teaching ...". $87 \mathrm{Vgl}$. Roy, O., Weg 327. Bei Karic geschieht das jedoch in einem weitaus offeneren Sinne als bei Fundamentalisten, die ebenfalls an einer Re-Universalisierung der Religion arbeiten.

88 Vgl. Karic, E., Bosnia 86; E. Karic, Land 65. 
z.B. der Muslime in Europa entgegen. Eine Leugnung des lokalen Profils des bosnischen Islams ist bei Karic damit nicht bezweckt, vielmehr kann „universal Islam“ im Kontext arabisierender Tendenzen in Bosnien verstanden werden, von denen sich Karic klar absetzt. Trotz aller Betonung des Universalen ist Karic demnach entschieden von seinen lokalen Kontexten und Traditionen geprägt. ${ }^{89}$ Bei Ramadans Universalisierung spielt das Lokale letztlich eine noch geringere Rolle als bei Karic, auch wenn seine ideengeschichtliche Prägung den Lesern nicht verbogen bleibt. ${ }^{90}$

Trotz starker Betonung des Universalen werden beide Ansätze in der Praxis angesichts der Fragmentierung und Pluralisierung der Muslime $^{91}$ möglicherweise auf Widerstand stoßen und können als Versuche angesehen werden, an einem Ideal einer universalen Einheit festzuhalten. Möglicherweise führt die negative Bewertung von Meinungsverschiedenheiten im Koran (vgl. Sure 2,113.213; 3,19; 23,59 u.ö.) dazu, dass im Islam, der als „religion of unity“92 bezeichnet werden kann, der Lokalisierungswiderstand besonders stark ausgeprägt ist. Allerdings hat es in der Praxis immer eine innere Pluralität („muslim diversity“) gegeben - angefangen von den Rechtsschulen und unterschiedlichen Lesarten des Koran bis hin zu Inkulturationen in verschiedenen lokalen Kontexten. ${ }^{93}$

89 Vgl. Balić, S., Das unbekannte Bosnien. Europas Brücke zur islamischen Welt, Köln/Weimar/Wien 1992, 349-353. Dass sein Konzept von Universalität sich klar von dem arabischer Muslime unterscheidet, betont Karic an anderer Stelle: „de même que les Arabes ont leurs manières traditionnelles de vivre l'universalité de l'islam, nous avons les nôtres“" (zit. nach X. Bougarel, définitions 5). Vgl. auch Karic, Enes, Die klassische und zeitgenössische Hermeneutik des Korans im Licht neuerer Rezeptionstheorien, in: Synthesis philosophica 4 (1989), 67-90, 88, wo von „mehrere(n) lokalen Theologien“ und einem „Theologienpluralismus“ die Rede ist. 90 Vgl. Ghadban, R., Ramadan 77.

91 Siehe oben Kapitel 4.

92 Waardenburg, J., Muslims 349.

93 Vgl. dazu Paret, R., Innerislamischer Pluralismus, in: Haarmann, U./

Bachmann, P. (Hg.), Islamische Welt zwischen Mittelalter und Neuzeit, Beirut 1979, 523-528 ausgehend von dem ab dem 8. Jahrhundert überlieferten Satz: „Die Meinungsverschiedenheit der Gemeinde ist Gnade." Vgl. auch Manger, Leif, Muslim Diversity. Local Islam in Global Contexts, in: Manger, L., Diversity, 1-36, 18: „This further strengthens the notion that Islam comprises not only the cosmological theme 
Während der Ansatz von Karic bislang außerhalb Bosniens fast überhaupt nicht rezipiert wurde, ${ }^{94}$ wird Ramadan in erster Linie von Nichtmuslimen teilweise sehr kritisch bewertet. Ralph Ghadban kommt zu folgendem Urteil über Ramadan: „Er versucht, die Moderne zu islamisieren: Die linken Werte der Emanzipation, die christlichen ethischen und spirituellen Werte und die humanistischen Werte, die er während seines Engagements mit kirchlichen und humanitären Organisationen erworben hat, erklärt er ohne Begründung zu einem Bestandteil des Islam. “" ${ }^{\text {95 }}$ Teilweise ist die Kritik an Ramadan sicherlich berechtigt. So deutet Ramadan die Nähe zur Befreiungstheologie nur schlagwortartig an, ohne etwa entsprechende Werke $\mathrm{zu}$ zitieren oder explizit Anregungen aus der Befreiungstheologie aufzugreifen. ${ }^{96}$ Seine prinzipielle Opposition gegen Globalisierung gerät möglicherweise in einen Widerspruch zur dialektischen Beziehung zur Gesellschaft, die er immer wieder einfordert. Gerade für traditionelle Muslime könnte Ramadan jedoch als Brückenbauer fungieren, dessen differenziert argumentierendes Konzept sich wohltuend von einem sich nur in den Kategorien „erlaubt“ und „verboten“ abspielenden religiösen System abhebt. ${ }^{97}$ Positiv zu bewerten ist ferner, dass Ramadan die Muslime auffordert, sich in die Gesellschaft einzubringen, anstatt sich in islamischen Binnenräumen abzuschotten. So charakterisiert Ammann Ramadan auch als „konservativen Reformer“98, der aufgrund seiner Herkunft auch für konservative Muslime glaubhaft sei.

of the holy texts, but lived identities in local contexts, emerging within ongoing debates about what is right and what is wrong." Ähnlich auch E. Karic, Hermeneutik. 94 Ausnahme ist der französische Balkanologe Xavier Bougarel (X. Bougarel, définitions).

95 Ghadban, R., Ramadan 63. Vgl. 33: „Ramadan will Europa islamisieren, die Herrschaft des Islam errichten."

96 Vgl. Ramadan, T., Muslims 199 und Ramadan, T., Globalisation 100, 120.

97 Anders z.B. Zaidan, A.M.A., Al-'Aqida. Einführung in die Iman-Inhalte, Offenbach 21999.

98 Ammann, L., Ramadan 31. 


\section{Konsequenzen: Interkulturelle Theologie im Globalisierungsdiskurs}

Die Überlegungen haben gezeigt, dass Globalisierung und Lokalisierung zentrale Begriffe sind, um den aktuellen muslimischen Diskurs in Europa zu verstehen. Analysen weiterer muslimischer Positionen sind notwendig und vielversprechend.

Die islamisch-theologische Diskussion ist von einer kritischen Auseinandersetzung mit Globalisierung geprägt, die ihr mit christlicher Theologie gemeinsam ist. ${ }^{99} \mathrm{Zu}$ beiden hier vorgestellten Positionen ließen sich auch vergleichbare christliche Positionen finden. Zumal angesichts des Themas ist folglich eine interkulturelle und interreligiöse Perspektive angebracht. Daher ist abschließend anzudeuten, welche Konsequenzen und Aufgabestellungen sich daraus für eine interkulturelle Theologie ergeben: ${ }^{100}$

(1.) Notwendig sind zunächst interreligiöse „Ent-Globalisierungen“ des Islamdiskurses. ${ }^{101}$ Ein Gesamtbild des Islam ergibt sich als Mosaik lokaler Erfahrungen. Das von Ramadan und Karic betonte negative Islambild der Medien hat seinen Grund darin, dass diese lokalen Erfahrungen zuwenig beachtet werden. Als Gegengewicht wären positive Darstellungen lokaler (Islam-)Kulturen hilfreich. ${ }^{102}$ Damit sollte eine Förderung lokaler Strukturen der Muslime einhergehen, ${ }^{103}$ denn die Medien nehmen eine auch in der

99 Vgl. z.B. Collet, G., Le christianisme - produit dérivé ou contre-courant de la globalisation. Observations et remarques dans une perspective européenne occidentale, in: LV(F) 58 (2003) 77-87; Schreiter, R., Globalisierung 151-158. 100 Als eine allgemeine Darstellung von grundsätzlichen Aufgaben der interkulturellen Theologie angesichts von Globalisierung vgl. Küster, V., Globalisierung ist ein Thema der Interkulturellen Theologie!, in: Vögele, W./ Wegner, G. (Hg.), Religion und Globalisierung, Rehburg-Loccum 2002, 39-46, $42 \mathrm{f}$.

101 Vgl. auch Mitri, T., Dialogue between Christians and Muslims Today, in: Ipgrave, M.(Hg.), The Road Ahead. A Christian-Muslim Dialogue, London 2002, 102-109, 108.

102 Vgl. dazu Schmid, H., Verhältnis 78f.

103 Dazu gehört neben einer Anerkennung islamischer Organisationen vor allem auch 
muslimischen Kommunikation noch verbreitete Importstruktur auf. Der Fokus auf das Lokale darf jedoch nicht umgekehrt dazu führen, globale Zusammenhänge aus dem Auge zu verlieren. So ist die Situation in anderen Ländern und Kontexten auf faire Weise auch in lokale Diskurse einzubringen. Man kann somit von der Notwendigkeit der Verschränkung beider Bewegungsrichtungen - vom Globalen zum Lokalen und umgekehrt - sprechen.

(2.) Globalisierung sollte nicht allein westlich definiert werden. Ansonsten wird eine islamische Gegenglobalisierung provoziert, die auf vielfältigen Ausgrenzungserfahrungen beruht. Es braucht vielmehr eine Globalisierung, die Raum lässt für verschiedene Religionen, auch für den Islam. ${ }^{104}$ Dazu muss die interkulturelle Kompetenz aller am Diskurs Beteiligter gestärkt werden und es müssen möglichst universal gültige ethische Standards der Kommunikation und Kooperation entwickelt werden.

(3.) Die Positionierung der Muslime hängt nicht nur oder primär von ihnen selbst ab, sondern auch, wie man sich in den westlichen Gesellschaften ihnen gegenüber öffnet. Folgende Wechselwirkung entsprechend auch der Dynamik der Globalisierung ist $\mathrm{zu}$ beobachten: Je weniger Beteiligungsraum Muslimen in Europa gewährt wird, desto stärker reagieren sie mit weiterer Abgrenzung. Die Polarisierung des Islam als der schlechthin „Andere" in Europa sollte aufgebrochen werden; der Islam als Bestandteil Europas ist zu beschreiben und zu erforschen - mit Konsequenzen bis in Lehrpläne und Studiengänge. ${ }^{105}$ Dies kann letztlich zu einem gelasseneren Umgang mit dem Thema Islam führen.

(4.) Schließlich können Muslime gemeinsam mit Christen kritische Impulse gegen die Globalisierung setzen und gemeinsam den Opfern der Globalisierung eine Stimme geben. Dieser 
gemeinsame Einsatz auf der Basis von Menschenwürde und Gemeinwohl ${ }^{106}$ ist in einer kritischen Distanz zur Globalisierung möglich, ohne dass daraus eine Totalopposition entstehen muss, welche die Religionen letztlich von der Gesellschaft entfremden würde. $\mathrm{Zu}$ einem solchen Engagement sind Netzwerke als neue inner- bzw. interreligiöse Allianzen erforderlich. ${ }^{107}$ Diese Netzwerke sollen durchaus lokal ansetzen, aber sich überregional verbünden, um auch politisch wirksam werden zu können. Dabei ist wichtig, dass Muslime Mitgestaltende sind, nicht nur in ein christliches Konzept Eingeladene. Ansonsten besteht die Gefahr, dass auch auf dieser Ebene im Kontext der Globalisierung vielfach erfahrene Muster der Ausgrenzung reproduziert werden.

106 Vgl. dazu Leirvik, O., Global Ethics and Moral Disagreement after September 11, 2001. A Christian-Muslim Perspective, in: Studies in Interreligious Dialogue 13 (2003), 18-29, 27.

107 Als Beispiel dafür können christlich-islamische Gesellschaften genannt werden (vgl. dazu http://www.kcid.de). Auf wissenschaftlicher Ebene ist z.B. GRIC (Groupe de Recherches Islamo-Chrétien) zu erwähnen (vgl. http://www.gric.asso.fr/). 